OPEN ACCESS

Received: 21.01.2021

Accepted: 29.01.2021

Published: 23.02 .2021

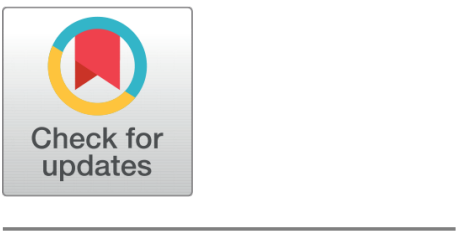

\section{Unified energy efficient distributed network management protocol to forward the data packets and to improve QoS in Wireless Sensor Networks}

\author{
N C Sachithanantham ${ }^{1 *}$, V Jaiganesh ${ }^{2}$ \\ 1 Research Scholar, PG \& Research Department of Computer Science, Dr.N.G.P Arts and \\ Science College, Coimbatore, 641048, Tamilnadu, India. Tel.: +91 9786595599 \\ 2 Professor, PG \& Research Department of Computer Science, Dr.N.G.P Arts and Science \\ College, Coimbatore, 641048, Tamilnadu, India
}

Citation: Sachithanantham NC, Jaiganesh V (2021) Unified energy efficient distributed network management protocol to forward the data packets and to improve QoS in Wireless Sensor Networks. Indian Journal of Science and Technology 14(6): 547-557. https://d oi.org/10.17485/IJST/v14i6.128

* Corresponding author.

Tel: +91 9786595599

sachinphd2020@gmail.com

Funding: None

Competing Interests: None

Copyright:

(c) 2021 Sachithanantham \& Jaiganesh. This is an open access article distributed under the terms of the Creative Commons Attribution License, which permits unrestricted use, distribution, and reproduction in any medium, provided the original author and source are credited.

Published By Indian Society for Education and Environment (iSee)

ISSN

Print: 0974-6846

Electronic: 0974-5645

\section{Abstract}

Background: Enhancing quality of service in forwarding the data packets from source to destination in WSN is a major task in contemporary networks. Everywhere the usage of WSN is increasing in the real world. Data sensing, data capturing and data forwarding is a routine process in modern era. Some of the dynamic problems arise as link failure, routing issues, packet delay etc that lead to the entire network shutdown. Hence, there is a need for development or enhancement of the protocol that finds the finest route to forward data packets in an optimal way. Objectives: The main objective of this study is to propose a unified energy efficient protocol to minimize the delay which will result in successful delivery of data packets from source to destination to improve the quality of service in WSN. Methods: This study proposes Unified Energy Efficient Distributed Network Management Protocol (UEEDNMP) focuses mainly on forwarding the data packets and finite routing to minimize the end to end delay. Parametric search technique along with CAFSM method is utilized to design the proposed protocol, to meet out the objective by forwarding the packets with better path from source to destination. Findings: NS2 Version 35 simulator is used to calculate the performance of the proposed UEEDNMP protocol. The simulation result shows UEEDNMP outperforms the existing protocols EH-WSN and ECO-LEACH in terms of data arrival rate, packet drop ratio, packet delivery ratio, energy consumption, network traffic, delay and network lifetime. Novelty: Comprehensive analyses signify that UEEDNMP has greater performance in forwarding the data packets with the finest routing in distributed networks, minimizing energy consumption, minimize the end to end latency time and maximize the life span of the network.

Keywords: Energy efficient protocol; delay; wireless sensor networks; data forwarding 


\section{Introduction}

The wireless sensor nodes deployed in unattended environment where the environment conditions vary significantly that leads to change in quality of sensor node due to connection and routing failure. The end to end delay of the sensor node depends on the stability of node that transmits data from source to destination. On the other hand, sensor nodes are compact devices that are provided with minimal resources, and extending the lifespan of the network is a great concern. Normally the sensor nodes are non-replaceable once deployed in a specific region. Since the sensors are running periodically by sensing and capturing the data's and forwarding to the destination. The nodes are battery power-driven, and it is not easy to replace or revive the battery all of a sudden. Due to the limited energy, sensor nodes drain their energy quickly, and lead to coverage problems of sensing area and fail to forward the captured data to neighboring node. Hence, energy conservation and packet delivery becomes an important concern in WSNs. Here the routing process indicates sharing of routes and searching the finest routes by the nodes to deliver the data packets in an optimal way with the help of routing protocols. Some of the characteristics of routing such as sharing of route information, maintaining the dynamic failure and identifying the optimal path etc. In recent years many energy efficient protocols are identified to forward the data packets efficiently and to increase the life span of network ${ }^{(1)}$ but since due to overload the enhancement of network lifetime becomes challenging task. In other hand MAC techniques and comprehensive sensing are utilized to identify the energy efficient protocols ${ }^{(2-4)}$. Subsequent study has been made to analyze the protocol and network and it reveals that the sensing communication consumes more energy, as well as forwarding the packets and transmitting to other nodes takes almost $75 \%$ of its total energy consumption, while the count of transmissions depends on the routing strategy followed in the network ${ }^{(5)}$.

Many routing protocols are proposed for dynamic and efficient routing in the distributed network environment ${ }^{(6)}$. Tree based routing ${ }^{(7)}$ also proposed in order to forward the packets with the help of parent node where all the packets are sent to the point where the parent is located, but the design of variable metric has become an issue in identifying the parent node by other nodes in the search space. The link quality in the transmission is also evaluated by using the expected data forwarding count that shows the loss of link between two edges to process for retransmission ${ }^{(8)}$. Energy efficient interruption identification framework (EEIDS) ${ }^{(9)}$ approach is proposed and executed. In EEIDS, Bayesian methodology is usually utilized for the hope of sensor nodes' electricity utilization by employing sensor nodes prior files that leads to prediction of energy during the data forwarding by using markov chain type. A different hop energy-efficient group protocol named MHEECA is proposed to obtain the best way between $\mathrm{CHs}$ in addition to BS for facts transmission using Dijkstra's algorithm ${ }^{(10)}$. Packet forwarding and distribution of the data may be non-uniform due to irregular movements happening in the heterogeneous network space ${ }^{(11)}$. Numerous routing techniques have been developed to maximize the energy efficiency and packet delivery ratio of WSNs, covering almost all layers of communication protocols ranging from the bottom to top layers in the network ${ }^{(12,13)}$. Improved versions of LEACH, proposed based on, selection of $\mathrm{CH}$, clustering, and packet forwarding. The selection of $\mathrm{CHs}$ is centric and performed by base station in the network based on the remaining energy available and node location ${ }^{(14)}$. A huge number of sensor nodes are deployed in a sensing area and formulate the process of identification of sink node to forward the packets to the destination. In this it is proved that there is only one sink node or parent node to deliver the packets to the destination by collecting data from all the neighboring nodes, but due to dynamic link failure the packets are not forwarded to the parent node and leads to unreliable connection ${ }^{(15)}$. In order to maximize the network lifespan, OPRP ${ }^{(16)}$ was proposed for WSN to take care of instantaneous routing optimization problems. It operates by measuring the static routes available and picking them by testing the time available. The findings reveal that it takes more time for the protocol to send the packet to the destination, where it implicitly means that the network lifespan is shortened. For WSN, Efficient Sensor Routing ${ }^{(17)}$ was proposed to preserve routing in a network that changes topology. Routing reliability was lost with the use of blind routing. Flooding, network congestion, and shortened network lifespan have arisen from the blind forwarding technique. Energy efficient underwater routing protocol was proposed ${ }^{(18)}$ to enhance the QoS in wireless sensor networks through cluster based multi path efficient routing, but due to dynamic connection failure the protocol does not suit for more packet delivery in a wide range. $\operatorname{IEERP}^{(19)}$ routing is designed to discover the finest route for efficient packet forwarding in an optimal way. Due to searching more routes the node spent more time in selecting the finest route and leads to more energy consumption and fails to perform well. RABCP ${ }^{(20)}$ was proposed to detect the dynamic link failure and find the alternate route from source to destination. IEE-LEACH is proposed ${ }^{(21)}$ for electing the $\mathrm{CHs}$ in the search space and employs all communication techniques such as single, multi, hybrid to further improve the energy efficiency of networks. EH-WSN and ECO-LEACH is proposed for best energy harvesting method and to forward the data packets efficiently, but due to poor congestion aware and end to end delay the network fail to receive the packets forwarded by sink ${ }^{(22)}$.To perform periodical operations by the node in the search space or networks the protocols should meet the following requirements 
- Efficient Data Forwarding: Forwarding data's to CHs or parent node without any noise to maximize the packet delivery ratio.

- Dynamic Routing: Efficient minimal routing is required to save energy and maximize the node search for efficient data forwarding to the upper layer.

- Minimization of Delay: Minimizing end to end delay is necessary for successful packet delivery and to enhance the lifetime of the network.

- Reconstruction: The protocols should have the capability of route reconstruction based on the data capturing and it should have its own frequency to connect and disconnect.

Various protocols are been identified to overcome the issues in EH-WSN and ECO-LEACH, but all the protocol metrics are concentrated based on the node counts and throughput and not concentrated in finding the finest path and minimize the end to end delay in routing process in the distributed WSN search space. This research work aims to develop a new protocol to: i) minimize end to end delay in searching the node. ii) forwarding packets in efficient manner and iii) minimizing the energy, packet drop ratio and maximizing the lifetime of the network.

\section{Proposed Methodology}

The efficient data forwarding and packet delivery model of the proposed UEEDNMP protocol is introduced. Unlike the LEACH, EH-WSN protocol here the nodes are assigned with different initial energy. Normally the nodes in the clusters forward the packets to the parent node and collectively the parent node will forward packets to the destination. Here the nodes will identify the nearest cluster head in the nearest neighboring cluster by using the nearest neighbor algorithm and forward the packets. If the energy gets drained, the nearest node will act as a parent node and receives the packet and forward to the CHs. Assume that there are $\mathrm{N}$ nodes employed in $\mathrm{R}$ region and UEEDNMP protocol works with multi techniques here, i) node catch model, ii) the nearest node discovery model and iii) multipath model.

When the destination is very close to the node the protocol follows node catch model by using parametric search technique to find the BS and forward the data packets. Node energy loss is denoted by E and the node catch denoted by NC.

Thus the parametric search technique as follows and given in the below equation,

$$
N C=\mathrm{N}^{n-1}=\sum_{R=0}^{N p u s h}\left(\begin{array}{c}
n \\
r
\end{array}\right) E^{p o p} R^{n-1}
$$

If the BS is near, NC is performed and the paths been identified. Where the node will search the nearest neighbor and $\mathrm{k}$ is the number of clusters in WSN and average node of the clusters are denoted by $\mathrm{N}_{D A}$ where the consumption of energy is calculated by calculating the distance between the source and destination. This suggests that not all nodes in the WSN share in the creation of clusters. By comparing all the signal strength of the packets forwarded by the $\mathrm{CHs}$, a node closest to the $\mathrm{CH}$ chooses to join the cluster and $\mathrm{CH}$ transmits the highest signal to the nodes and here the node catch model is implemented. Determines to be closer to the $\mathrm{CH}$ after each node it needs to notify the $\mathrm{CH}$, which $\mathrm{CH}$ will join, that it has become a member node of this cluster. Each node near the $\mathrm{CH}$ sends a request message to the chosen $\mathrm{CH}$, which includes the identity of the node and the identification of the $\mathrm{CH}$.

In the nearest node discovery model finding the closest cluster to forward the packets is the main objective and here UEEDNMP follows optimal search technique and greedy search method to identify the nearest cluster in the search space. Direct computation algorithm also used here to compute the node positions and distance from source to destination. dy and $\mathrm{dx}$ are termed as source and destination distance in the search space.

$$
\frac{d y}{d x}=\sum(x j-x i j) \frac{y}{x} N C^{c o m p}=\int_{j=1}^{M} U p o p^{j} \times M \quad d y
$$

Where $\mathrm{x}, \mathrm{j}$ represents the computational methods and $\mathrm{NC}$ comp termed as nearest node discovery. With the help of the above equation the nearest node distance is calculated. The coverage updation of the entire network is calculated using,

$$
\varnothing^{h+1}=\varnothing^{h} / \exp \left(N C^{\varnothing} * c^{\varnothing}\left(o^{\varnothing * h+\frac{1}{F}: M(0,1)}\right)-1\right)
$$


Where the closest clusters distance is calculated to check the proximity value and to classify a new example $\mathrm{x}$ by finding the training by finding the training example $\mathrm{h} x \mathrm{xi}$, yi $\mathrm{i}$ that is nearest to $\mathrm{x}$ according to Euclidean distance. It will be good to deploy further nodes across the base station if it is under the coverage area. As mentioned there is generally reasonable coverage efficiency for a randomly and uniformly deployed WSN. In comparison, over the long term, one static base station could be unable to gather data effectively. Configuring several clusters in BS so that nodes can send data to the nearest one is an intuitive approach, or base stations take turns operating to coordinate the energy consumption between all nodes.

The local clusters in WSN can be computed with the help of the below equation,

$$
D^{\pi}=\left(\pi^{x}+1\right) \frac{d x}{d y} N^{i+1} D^{h+1}=\left(1+D^{i+1}+D^{\pi}\right) D^{h}
$$

Where, the proximity metrics can be calculated based on the source and destination distance. That would mean that ties to nodes are deemed more important since they have a greater radius that reduces side paths near to the periphery of the artery.

- Various stages involved in UEEDNMP protocol are,

- Identifying the Cluster Head

- Cluster Head Selection

- Cluster Configuration using CAFSM

- Finest Route Selection

- Data Forwarding in Distributed Network

In the real time, UEEDNMP initially search for the nearest cluster and check for the energy from the available nodes. Later the distance will be calculated between the source node and the $\mathrm{CH}$ which is found newly and the finest route will be selected during the forwarding process by the protocol using the ArcGIS analysis to after analyzing the route layer, service area, closest $\mathrm{CH}$ and position of nodes in WSN. After the analysis the forwarding process will be done by the protocol using CAFSM method.

\subsection{Identifying the cluster head}

During the search of $\mathrm{CHs}$ by the source node, if the head of the cluster leaves the cluster, then new head is assigned to each fragment if the cluster is destroyed by loss of energy. Relocation of cluster head works as follows. Assuming that the cluster head has access to the most recent information on the location of the objective with respect to each node of the cluster, it will preserve a truthful list of the best applicants for the cluster head. When the cluster head wishes to leave the cluster, it sends a message containing a filtered list of the best candidates for the cluster head to its neighbors. There the node will identify the $\mathrm{CHs}$ and passes the information. Hierarchical parametric search method is followed here is shown in the equation.

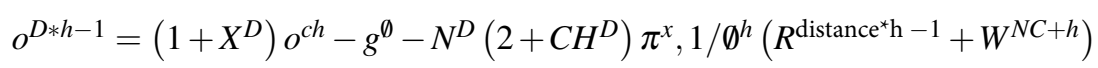

Where, $\mathrm{CH}$ is cluster head, $\mathrm{N}$ is the node and $\mathrm{R}$ is ratio of nodes and distance calculated and $\mathrm{W}$ is the weightages of node. Finally, Closest CHs are identified.

\subsection{Cluster head selection}

After identifying the closest CHs there is a need of selection of CHs to proceed for data transmission to the parent node. The path selection is rendered by gathering detailed information from the chosen clusters about the distance value, the amount of energy available and the time, etc. It also includes the specifics of the path mug identification. Once the data is gathered for improved route selection for $\mathrm{CH}$ s data forwarding, packets for ready state are obtained by a sink node. Consider P1, P2, and P3 as data with separate node numbers available to reach the destination node from the nearest parent node of the $\mathrm{CHs}$, and $\mathrm{P}$ as routes for $\mathrm{N}$ to reach the destination node. After the operation of selection of CHs is over, the nodes' energy is determined to forward the data packets.

\subsection{Cluster configuration using CAFSM}

Multiple metrics are used by the congestion-aware forwarder selection method (CAFSM) to select the next hop node in the search space. The expense parameter of this method is inversely proportional to the improvement that can be accomplished in the node selection and $\mathrm{CH}$ selection. Although the destination is linearly proportional to the neighbors' congestion rate, the direction of routing, and the energy required to deliver a packet. The progress made to the destination is also more influential than the other metrics, allowing the distance between the current nodes of sending packets. 
Here the wait time is calculated and termed as WT. The CAFSM method is implemented using the below equation,

$$
P^{i}=1-\left(1-\left(n_{n}\right)\right) N^{x d s}(n j) / N^{\text {wait }} x \frac{R^{\text {distance } * h-1}}{\text { Total Distance }} X^{i j}
$$

Where the term $\mathrm{P}$ denotes the performance of the node and the wait is calculated to forward the packets to the CHs parent node that is identified earlier using equation 5.

\subsection{Finest route selection}

Once the identified $\mathrm{CH}$ is configured using CAFSM the route selection needs to be started between the nodes for efficient data transmission. Here Minimum Transmission Power Routing (MPR) method is utilized to select the optimal route to forward the packets. It selects the path that uses minimum energy consumed by the nodes and the length 1 is calculated. The total energy of the route $\mathrm{T}_{i}$ is computed by the following equation,

$$
(P)^{i}=\sum_{i=0}^{p-i(\text { distance })}(P) n^{k} l^{n-1}
$$

Finally, the path selection is done and the protocol will send the ready message to make sure that it is ready for receiving the packets from the source. MPR will achieve the energy consumption of the total network however the residual energy will not be considered for transmission process. The path selection is chosen the below strategies,

- Node energy level

- Transmission power

- Previous transmission rates

- Total energy of $\mathrm{CH}$ in the network

\subsection{Data forwarding in the distributed network}

Since the nodes are very much aware of their position and location in the search space each node has ability to use diverse frequencies for packet forwarding and packet receiving and it is imagined that the node has a bi-channel transceiver. Each node has capability to forward data packets in a longer distance. Here the new way of forwarding the packets to the destination have been imposed where the nodes contain different parameters such as distance, energy level and time from the $\mathrm{CH}$ node.

Consider a WSN comprising $\mathrm{N}$ wireless sensor nodes that are consistently distributed within a network size of $\mathrm{d} \mathrm{x} \mathrm{d}$ and within each cell one of the nodes acts as the cluster head $(\mathrm{CH})$ in the Base Station. Various states of $\mathrm{CHs}$ are transmitting, receiving and sleep state. Let's assume that $\mathrm{N}_{i e}, \mathrm{~N}_{t h}, \mathrm{~N}_{e n}, \mathrm{~N}_{\text {trans }}$, denotes initial energy, throughput, energy consumption and data forwarding time. The selection of the access is based on constraints such as the node distance, number of nodes close to a particular node, and the energy level which is calculated in the previous step. During every transmission time the intervals are calculated and the distance is used for calculation and the data is forwarded in the distributed network environment effectively. The $\mathrm{CH}$ data forwarding and energy is calculates using the below equation,

$$
N=\operatorname{Node} \frac{N^{i e}-N^{\text {th }}}{N^{\text {trans }}} N \sum_{i=0}^{\text {nodeselect }} \text { distance }(P) \text { node distance }
$$

\section{Performance metrics}

This research work makes use of the following metrics to analyze the performance of the proposed protocol UEEDNMP against EH-WSN and ECO-LEACH protocols as selected as the baseline protocols discussed above.

Data Arrival Rate

- Packet Drop Ratio - Measures the drop ratio of data packets during the data transmission

- Packet Delivery Ratio - Measures the successful packet delivery from source to sink

- Energy Consumption - Total number of energy consumed by the node

- Network Traffic - Measures the Traffic during the packet forwarding

- Delay - Time consumption by the protocol to deliver the packets

- Data Arrival Rate Analysis - Analysis of total Number of data arriving from source to destination

- Network lifetime - Measures the duration of the network and the process 


\section{Simulation settings}

This section discusses evaluating the performance of the UEEDNMP using NS2 network simulator. In common, there is no trusted simulator for evaluating protocols in the distributed network. This paper attempts to compare UEEDNMP against EHWSN and ECO-LEACH. This research work prefers the tool command language to use in the NS2 simulator. Table 1 shows the simulation setting used for evaluating the proposed UEEDNMP protocol.

Table 1. Simulation \& experimental eettings in NS2

\begin{tabular}{ll}
\hline Parameters & Values \\
\hline Nodes Count & $500-2500$ \\
Time Slot & $100 \mathrm{~ms}$ \\
Data Packet Rate & 0.5 packets \\
Initial Transmission Range of Deployed Nodes & $75 \mathrm{~m}$ \\
Type of Traffic & Wireless \\
Initial Energy of Each Node & $20 \mathrm{~J}$ \\
Sensing Range & $10 \mathrm{~m}$ \\
Threshold Distance & $75 \mathrm{~m}$ \\
Mobility Model & Random Way Point \\
Simulation Time & $5000 \mathrm{~s}$ \\
Data Payload & 2000 bit \\
\hline
\end{tabular}

\section{Results and Discussions}

\subsection{Packet drop ratio analysis}

Figure 1 represents the packet drop ratio against the node count during the forwarding process. It is clearly understood that the packet drop ratio is slightly increases when the node count increases. Here the packet drop ratio is minimized to $15 \%$ and an average of $21 \%$ in UEEDNMP protocol which is lower than the baseline protocols EH-WSN and ECO-LEACH. Packets are forwarded with a minimal drop ratio in the WSNs with the help of proposed protocol. It is noted that the proposed protocol performs better than the existing and delivered the remarkable output.

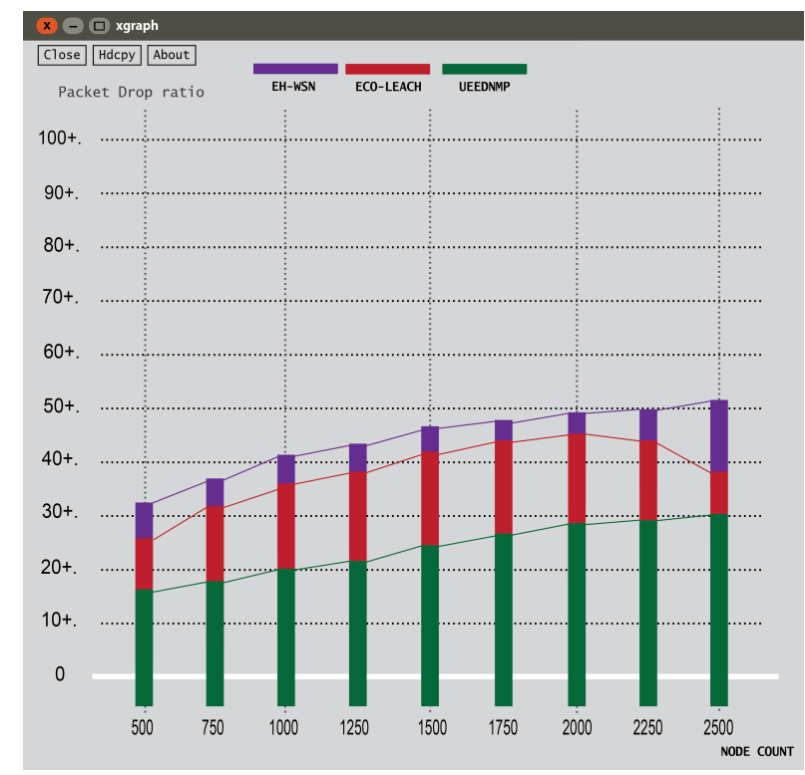

Fig 1. Packet drop ratio analysis 


\subsection{Packet delivery ratio analysis}

Figure 2 presents the packet distribution ratio against node count. It is noticeable that as the number of nodes is expanded for all protocols, the packet delivery ratio is slightly decreased. This is because an improvement in node count contributes to the condition of numerous path splits in the unsecured network, and thus there is degradation in transmitting the packets to the destination. The packet delivery ratio is maximized to $92 \%$ and an average of $86 \%$ in UEEDNMP protocol where the baseline protocols EH-WSN and ECO-LEACH has minimal ratio of delivery. It is trustworthy that, the proposed protocol performs better than the existing protocols.

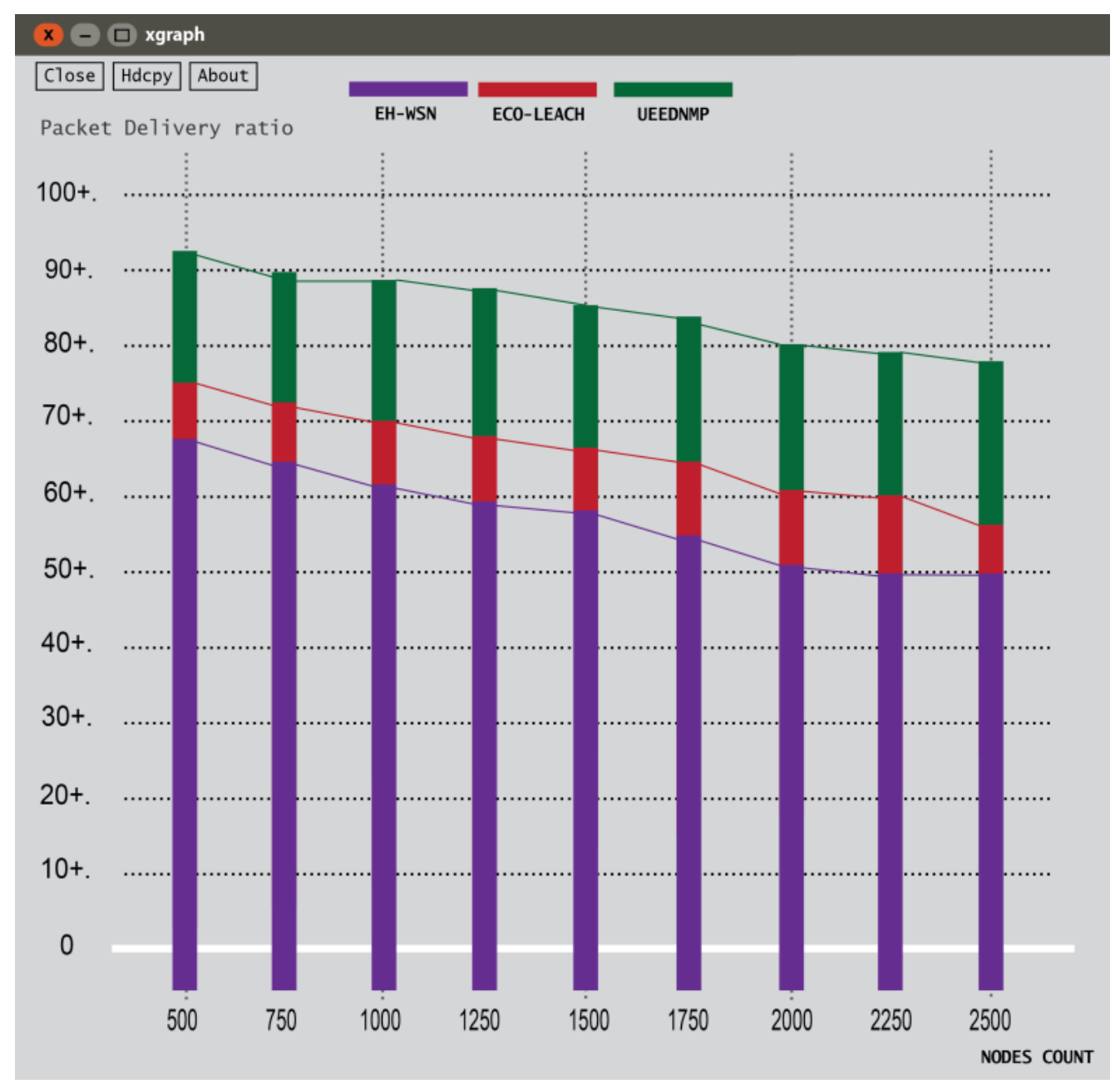

Fig 2. Packet delivery ratio analysis

\subsection{Delay analysis}

Delay analysis is presented in Figure 3 of the proposed protocol against the current protocols. It implies that the delay will be increased by an increase in node count. When testing the delay protocols, it was found that when node count increases, UEEDNMP still faces delay and found slight change, but after a certain amount, the delay is minimized. When studying EHWSN and ECO-LEACH protocols, it is clear that as node counts increase, the existence of delay tends to increase. The key explanation for this is that EH-WSN and ECO-LEACH chose the low energy node to forward the packet, resulting in route change and failure. 


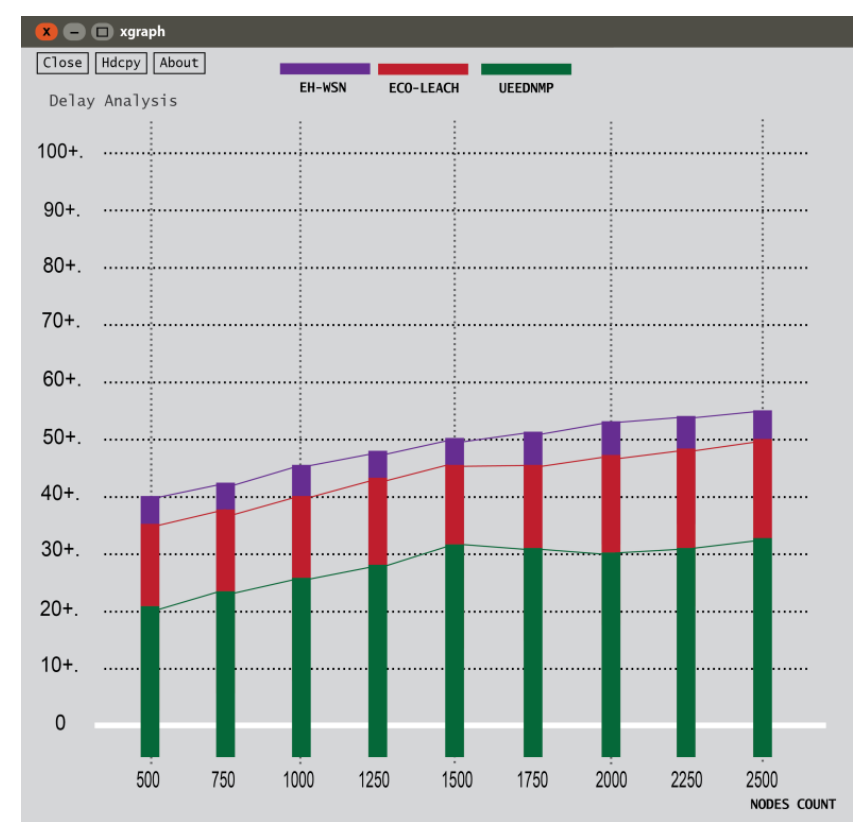

Fig 3. Delay Analysis

\subsection{Energy consumption ratio analysis}

Figure 4 presents the energy consumption ratio against nodes. It shows how much energy is worn out from one end to another during data transmission in WSN. It is indisputable that, as compared to EH-WSN and ECO-LEACH, the energy usage of UEEDNMP is significantly lower. High energy consumption suggests the protocol's inconsistency as the node count is raised. UEEDNMP absorbed only the appropriate energy spectrum (i.e. 18.1\%), but EH-WSN consumed 42\%, and ECO-LEACH consumed $42 \%$. This is due to the reconstruction of the incorrect path and the number of $\mathrm{CH}$ positions.

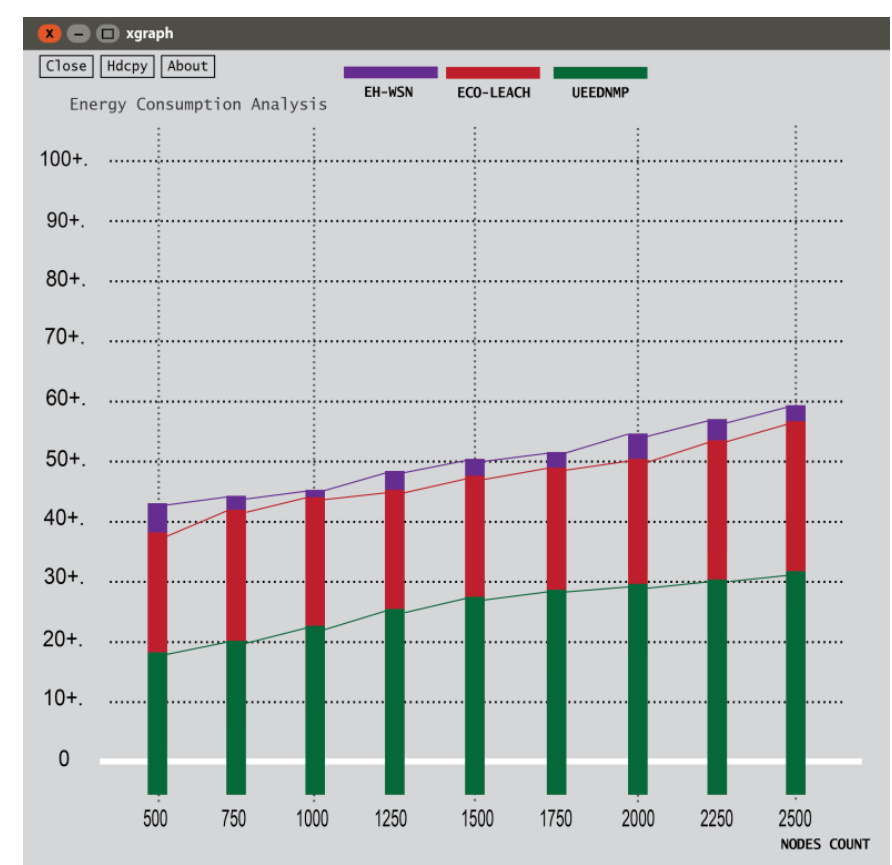

Fig 4. Energy consumption ratio analysis 


\subsection{Data arrival rate analysis}

Data arrival ratio is presented in Figure 5. It is noted that is how much time taken to deliver the data without any replication in the wireless sensor networks and also shows that how much noisy data captured and eliminated while broadcasting. In this analysis, it is clearly understood that the data delivery rate is high in UEEDNMP protocol when compared to EH-WSN and ECO-LEACH protocols since UEEDNMP protocol chooses the correct route rediscovering process during the energy depletion. The arrival rate is almost $93 \%$ and an average of $86 \%$ is been achieved.

\subsection{Network traffic analysis}

Network Traffic analysis is presented in Figure 6. It indicates the routing traffic during the data transmission or the amount of data transmitting at a given point of time. In this performance indicator the protocol UEEDNMP shows high rate in packet forwarding process even the load is high compared to the baseline protocols EH-WSN and ECO-LEACH. Since UEEDNMP uses the cluster head selection at the initial stage the data transmission rate is high compared to other protocols.

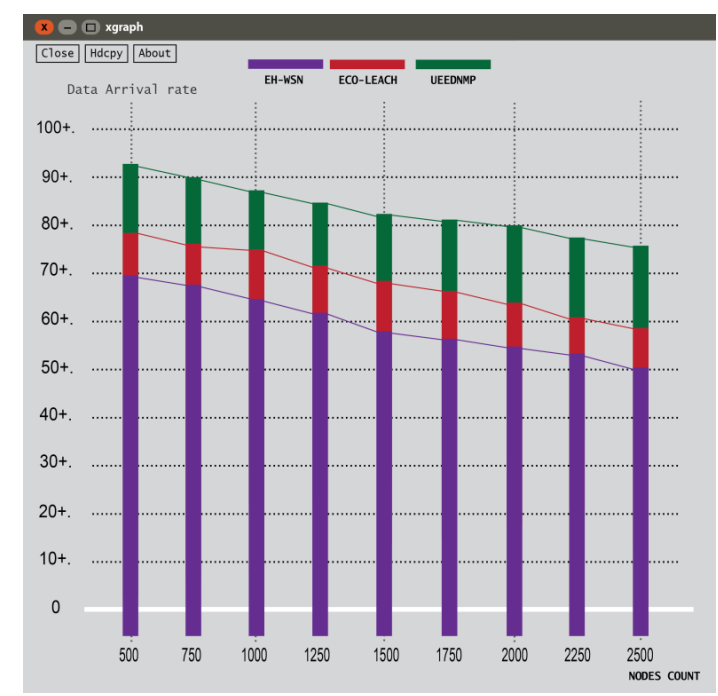

Fig 5. Data arrival rate analysis

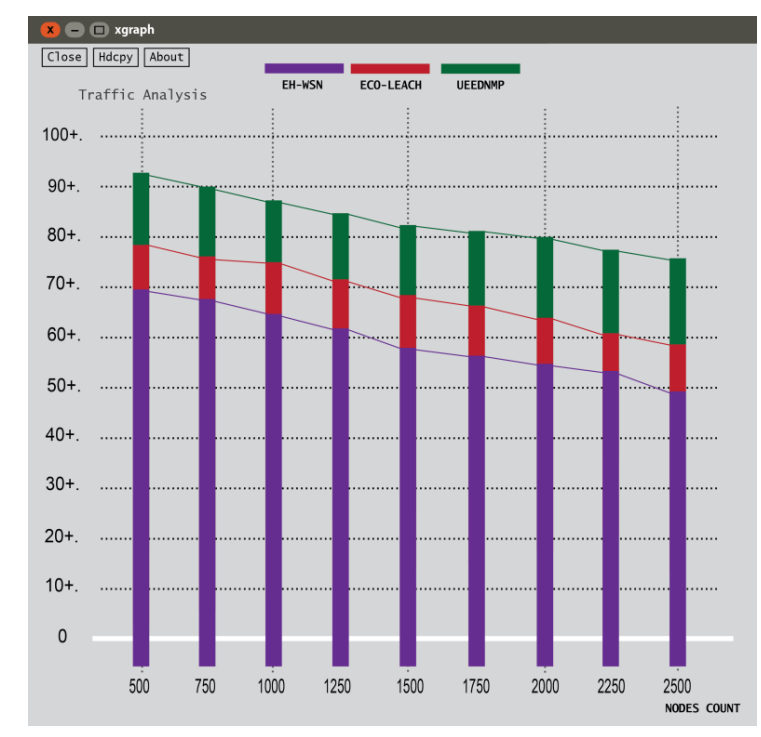

Fig 6. Network traffic analysis 


\subsection{Network lifetime analysis}

Figure 7 represents the network lifetime analysis. It shows that how long the network is functioning during the data transmission process and how far the nodes forwarding the data packets from one to other successfully. The lifetime of the network is maximized up to $94 \%$ with an average of $87 \%$ by using UEEDNMP where the baseline protocols EH-WSN and ECO-LEACH protocols doesn't show much performance in maximizing the network lifetime. It is trustworthy that the proposed UEEDNMP protocol made a remarkable performance in enhancing the network lifetime.

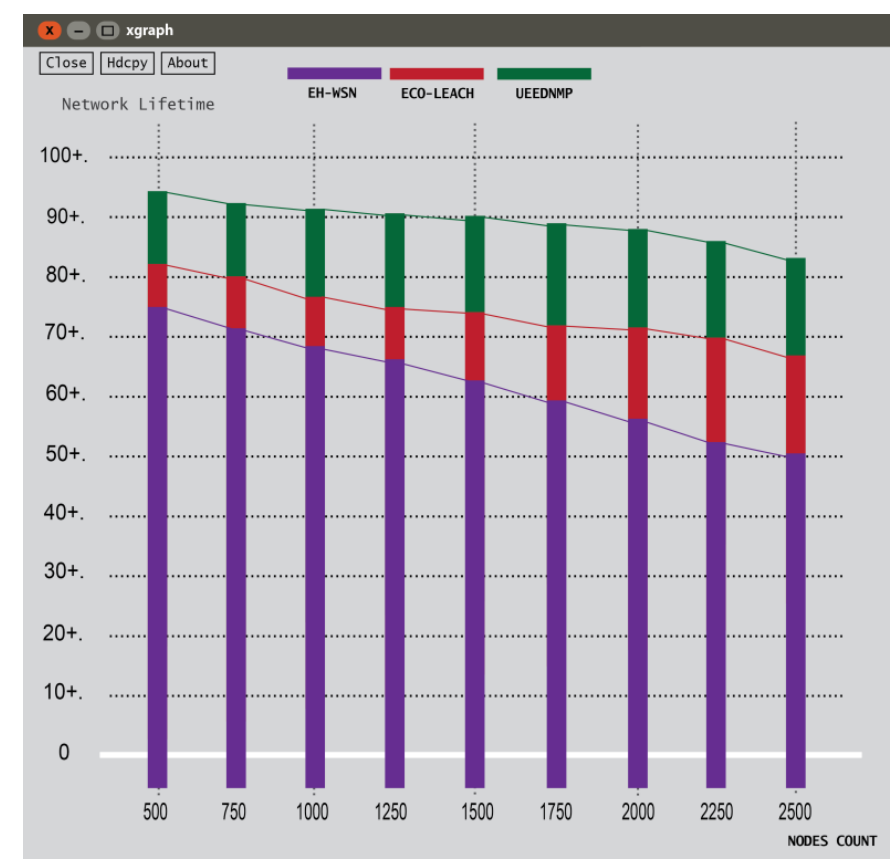

Fig 7. Network lifetime analysis

\section{Conclusion}

The study has proposed a novel protocol namely UEDDNMP to enhance the QoS in wireless sensor networks. The protocol has marked a great performance in terms of forwarding the data successfully and minimizing the end-to-end delay during the data transmission process. CAFSM and parametric search technique and MPR method are employed to ensure that the data is getting forwarded from source to destination. The proposed protocol performance has been evaluated using selected performance metrics in the network simulator. The simulation results shows that the proposed UEEDNMP protocol has tremendous improvement in performance based on data forwarding and end-to-end delay compare with EH-WSN and ECOLEACH protocols. The next work will examine the malicious and intruding nodes by applying the machine learning algorithms with different parameter.

\section{References}

1) Carrano RC, Passos D, Magalhaes LCS, Albuquerque CVN. Survey and Taxonomy of Duty Cycling Mechanisms in Wireless Sensor Networks. IEEE Communications Surveys \& Tutorials. 2014;16(1):181-194. Available from: https://dx.doi.org/10.1109/surv.2013.052213.00116.

2) Huang P, Xiao L, Soltani S, Mutka MW, Xi N. The Evolution of MAC Protocols in Wireless Sensor Networks: A Survey. IEEE Communications Surveys \& Tutorials. 2013;15(1):101-120. Available from: https://dx.doi.org/10.1109/surv.2012.040412.00105.

3) Younis O, Fahmy S. HEED: a hybrid, energy-efficient, distributed clustering approach for ad hoc sensor networks. IEEE Transactions on Mobile Computing. 2004;3(4):366-379. Available from: https://dx.doi.org/10.1109/tmc.2004.41.

4) Saleem M, Ullah I, Farooq M. BeeSensor: An energy-efficient and scalable routing protocol for wireless sensor networks. Information Sciences. 2012;200:38-56. Available from: https://dx.doi.org/10.1016/j.ins.2012.02.024.

5) Yan J, Zhou M, Ding Z. Recent Advances in Energy-Efficient Routing Protocols for Wireless Sensor Networks: A Review. IEEE Access. 2016;4:5673-5686. Available from: https://dx.doi.org/10.1109/access.2016.2598719.

6) Gnawali O, Fonseca R, Jamieson K, Moss D, Levis P. Collection tree protocol. Proc 7th ACM Conf Embedded Netw Sensor Syst. 2009;p. 1-14. Available from: https://doi.org/10.1109/ACCESS.2016.2598719. 
7) Shivaji SS, Patil AB. Energy Efficient Intrusion Detection Scheme Based on Bayesian Energy Prediction in WSN. In: 2015 Fifth International Conference on Advances in Computing and Communications (ICACC). 2015;p. 114-117. Available from: https://doi.org/10.1109/ICACC.2015.107.

8) Han L. A Multiple-Hop Energy Efficient Clustered Algorithm for Heterogeneous WSN. In: 2012 Fourth International Conference on Multimedia Information Networking and Security. 2012;p. 183-186. Available from: https://doi.org/10.1109/MINES.2012.36.

9) Shweta V, Kuma R. Variants of LEACH routing protocol in WSN: A comparative analysis. In: Proceedings of the 2018 8th International Conference on Cloud Computing. 2018;p. 11-12. Available from: https://10.1109/CONFLUENCE.2018.8442643.

10) Ke W, Yangrui O, Hong J, Heli Z, Xi L. Energy aware hierarchical cluster-based routing protocol for WSNs. The Journal of China Universities of Posts and Telecommunications. 2016;23(4):46-52. Available from: https://dx.doi.org/10.1016/s1005-8885(16)60044-4.

11) Heinzelman W, Chandrakasan A, Balakrishnan H. Energy-E_cient Communication Protocols forWireless Microsensor Networks. In: Proceedings of the 33rd Annual Hawaii International Conference on System Sciences. 2000;p. 4-7. Available from: https://10.1109/HICSS.2000.926982.

12) Arumugam GS, Ponnuchamy T. EE-LEACH: development of energy-efficient LEACH Protocol for data gathering in WSN. EURASIP Journal on Wireless Communications and Networking. 2015;2015(1). Available from: https://dx.doi.org/10.1186/s13638-015-0306-5.

13) Tan N. An Improved LEACH Routing Protocol for Energy-Efficiency of Wireless Sensor Networks. 2012. Available from: https://doi.org/10.6029/smartcr. 2012.05.006.

14) Biswas S, Morris R. Opportunistic routing in multi-hop wireless networks. ACM SIGCOMM Computer Communication Review. 2004;34(1):69-74. Available from: https://dx.doi.org/10.1145/972374.972387.

15) Mao X, Tang S, Xu X, Li XY, Ma H. Energy-efficient opportunistic routing in wireless sensor networks. IEEE Trans Parallel Distrib Syst. 2011;22(11):19341942. Available from: https://doi.org/10.1109/TPDS.2011.70.

16) An W, Ci S, Wu D, Han Y, Qi Y, Lin T. Transmission cost minimization with vulnerability constraint in wireless sensor networks. 2013 IEEE Wireless Communications and Networking Conference (WCNC). Available from: https://doi.org/10.1109/WCNC.2013.6554751.

17) Abbasi U, Awang A, Hamid N. A Cross-Layer Opportunistic MAC Routing Protocol to Improve Reliability in WSN. Proceedings of Asia-Pacific Conference on Communications. 2014;p. 36-41. Available from: https://doi.org/10.1109/APCC.2014.7091601.

18) Ahmed M, Soomro MA, Parveen S. Javed Akhtar1 and Nadeem Naeem3 CMSE2R: Clustered-based Multipath Shortestdistance Energy Efficient Routing Protocol for Underwater Wireless Sensor Network. Indian Journal of Science and Technology;12:1-8. Available from: https://10.17485/ijst/2019/v12i8/ 141788.

19) Helen D, Arivazhagan D. An Intelligent Energy Efficient Routing Protocol for Mobile Ad-Hoc Network. Indian Journal of Science and Technology. 2016;9(45):1-5. Available from: https://dx.doi.org/10.17485/ijst/2016/v9i45/104140.

20) Nithyanandh S, Jaiganesh V. Reconnaissance Artificial Bee Colony Routing Protocol To Detect Dynamic Link Failure In Wireless Sensor Network". International Journal of Scientific \& Technology Research. 2019;10(10):3244-3251. Available from: https://www.ijstr.org/final-print/oct2019/ Reconnaissance-Artificial-Bee-Colony-Routing-Protocol-To-Detect-Dynamic-Link-Failure-In-Wireless-Sensor-Network.pdf.

21) Liu Y, Wu Q, Zhao T, Tie Y, Bai F, M J. An Improved Energy-Efficient Routing Protocol for Wireless Sensor Networks. Sensors. 2019;19(20). Available from: https://doi.org/10.3390/s19204579.

22) Bahbahani MS, Alsusa E. A Cooperative Clustering Protocol With Duty Cycling for Energy Harvesting Enabled Wireless Sensor Networks. IEEE Transactions on Wireless Communications. 2018;17(1):101-111. Available from: https://dx.doi.org/10.1109/twc.2017.2762674. 